\title{
Characterization of the Streptomyces lividans PspA Response ${ }^{\nabla}$
}

\author{
Kristof Vrancken, Lieve Van Mellaert, and Jozef Anné* \\ Laboratory of Bacteriology, Rega Institute for Medical Research, Katholieke Universiteit Leuven, \\ Minderbroedersstraat 10, B-3000 Leuven, Belgium
}

Received 18 December 2007/Accepted 26 February 2008

\begin{abstract}
Phage shock protein (Psp) is induced by extracytoplasmic stress that may reduce the energy status of the cell. It is encoded in Escherichia coli by the phage shock protein regulon consisting of $p \operatorname{sp} A B C D E$ and by $p s p F$ and $p s p G$. The phage shock protein system is highly conserved among a large number of gram-negative bacteria. However, many bacterial genomes contain only a $p s p A$ homologue but no homologues of the other genes of the Psp system. This conservation indicates that PspA alone might play an important role in these bacteria. In Streptomyces lividans, a soil-borne gram-positive bacterium, the phage shock protein system consists only of the $p s p A$ gene. In this report, we showed that $p s p A$ encodes a 28-kDa protein that is present in both the cytoplasmic and the membrane fractions of the $S$. lividans mycelium. We demonstrated that the $p s p A$ gene is strongly induced under stress conditions that attack membrane integrity and that it is essential for growth and survival under most of these conditions. The data reported here clearly show that PspA plays an important role in S. lividans under stress conditions despite the absence of other psp homologues, suggesting that PspA may be more important in most bacteria than previously thought.
\end{abstract}

Bacterial cells need to be able to respond to a wide variety of extracytoplasmic stresses such as heat, osmotic, and alkaline stresses. A large number of extracytoplasmic stress responses have been identified (21), one of which is the phage shock protein ( $p s p)$ response. This $p s p$ system is highly conserved in a large number of gram-negative bacteria. In Escherichia coli, it consists of the divergently transcribed cistron $p s p F$, the $p s p A B C D E$ operon, and the more recently discovered $p s p G$ gene $(11,17)$.

The E. coli psp system is induced by a wide variety of stressful environmental conditions such as extreme heat shock $\left(50^{\circ} \mathrm{C}\right)$, ethanol treatment, hyperosmotic shock, and exposure to organic solvents or proton ionophores such as carbonyl cyanide $m$-chlorophenylhydrazone (CCCP) $(4,28)$. Furthermore, the $p s p$ response is induced by conditions or mutations that reduce the efficiency of the general $(\mathrm{Sec})$ and twin-arginine (Tat) secretion pathways $(7,9)$. On the other hand, when $p s p A$ is overexpressed, secretion via the Sec pathway becomes more efficient, and Tat-dependent secretion even shows a fourfold increase (7).

Although considerable research has been devoted to the $p s p$ response in several of the Enterobacteriaceae including Yersinia species, E. coli, and Salmonella species, rather less attention has been paid to its role in gram-positive bacteria.

Streptomycetes are soil-borne gram-positive bacteria that are well known for the production of a large variety of secondary metabolites. About two-thirds of the currently known natural antibiotics are synthesized by streptomycetes (26). Furthermore, it has proven to be a valuable alternative host for the

\footnotetext{
* Corresponding author. Mailing address: Laboratory of Bacteriology, Rega Institute, Katholieke Universiteit Leuven, Minderbroedersstraat 10, B-3000 Leuven, Belgium. Phone: 3216 337371. Fax: 3216 337340. E-mail: Jozef.Anne@rega.kuleuven.be.

${ }^{\nabla}$ Published ahead of print on 7 March 2008.
}

production of various heterologous proteins of biopharmaceutical and industrial importance $(20,22)$.

Recently, we have shown that the Streptomyces lividans PspA homologue stimulates the production of homologous and heterologous proteins secreted through either the Sec or the Tat pathway in this organism (25). It was remarkable to learn that in $S$. lividans PspA by itself could improve protein secretion, since $S$. lividans does not contain any of the $p s p B C F$ genes thought to be needed for a functional $p s p$ response (5). The PspB and PspC proteins are presumed to be the sensors of extracytoplasmic stress, and depending on the stress condition, a different protein is needed to obtain a $p s p$ response. Most inducing stimuli depend entirely on PspB and PspC (19). However, some stress conditions rely on only one of the $p s p B$ and $p s p C$ genes. Finally, some responses, like the induction of PspA by heat shock in E. coli, do not require PspB or PspC at all (27). The fact that $p s p A$ homologues are present in a large percentage of the sequenced bacterial genomes and the fact that most of them are not adjacent to any other psp genes (5) indicate that PspA may play an important role in bacteria even when PspB and PspC are not present.

In this study, we investigated the role of $S$. lividans PspA. Therefore, the expression pattern of the $p s p A$ gene was studied in wild-type (WT) $S$. lividans cells subjected to a variety of extracytoplasmic stresses. Furthermore, the importance of PspA was shown by subjecting a $p s p A$ mutant to the same stress conditions. The behavior of this mutant and the expression pattern provide evidence for a role for PspA in the extracytoplasmic stress response of S. lividans.

\section{MATERIALS AND METHODS}

Strains, media, and growth conditions. Streptomyces lividans TK24 and its derivatives were precultured in $5 \mathrm{ml}$ phage medium (15) supplemented with thiostrepton $(10 \mu \mathrm{g} / \mathrm{ml})$ or apramycin $(50 \mu \mathrm{g} / \mathrm{ml})$, if necessary, and grown at $27^{\circ} \mathrm{C}$ with continuous shaking at $300 \mathrm{rpm}$ for $48 \mathrm{~h}$. After homogenizing the mycelium, the strains were inoculated in NB medium $(10 \mathrm{~g} / 360 \mathrm{ml}$; International Diagnostics Group). When this NB medium was to be buffered, $40 \mathrm{ml}$ of morpholinepro- 
panesulfonic acid (MOPS) solution (0.5 M, pH 7.2) was added, resulting in NM medium (24). For solid medium, MRYE (2) was used and was supplemented with thiostrepton $(50 \mu \mathrm{g} / \mathrm{ml})$, if applicable.

Protoplast formation and the subsequent transformation of $S$. lividans were carried out according to procedures described previously by Kieser et al. (12). To test the distinct activities, precultures of $S$. lividans transformants grown in phage medium ( $48 \mathrm{~h}$ ) were used to inoculate $50 \mathrm{ml} \mathrm{NB} / \mathrm{NM}$ medium, and cultures were subsequently cultivated for 24 to $48 \mathrm{~h}$.

RNA isolation and RT-PCR. Total RNA was isolated from S. lividans strains as described previously by Van Dessel et al. (23). RNA concentration and purity were determined by measurement of the optical density at $260 \mathrm{~nm}\left(\mathrm{OD}_{260}\right)$ and by measurement of the ratio of the $\mathrm{OD}_{260}$ to the $\mathrm{OD}_{280}$, respectively. Samples used for reverse transcriptase PCR (RT-PCR) were also treated with DNase I (Qiagen) (final concentration, 0.5 units/ $\mu \mathrm{l}$ ) to remove any residual chromosoma DNA. RT-PCR was performed with $100 \mathrm{ng}$ of total RNA using the Access RT-PCR system (Promega). To investigate the presence of an operon structure encompassing the $p s p A$ gene, primers $2168 \mathrm{~F}$ (5'-ATGATCTTCCGCGCGAAG$\left.3^{\prime}\right), 2168 \mathrm{R}$ (5'-CTACTGCTTGTCGAAGCG-3'), 2169F (5'-CCGGGATTTTC CGGACAAC- $\left.3^{\prime}\right)$, and 2167R (5'-CGTCGCCGTTCTCCATCT-3') were used.

Extraction of membrane proteins. WT $S$. lividans TK24 was inoculated (2\%) in NM medium. After $24 \mathrm{~h}$ of growth, the mycelium was harvested by centrifugation, and the cells were resuspended in $50 \mathrm{mM}$ Tris- $\mathrm{HCl}(\mathrm{pH} 7.2)$ buffer. Following this resuspension, the cells were lysed in a French pressure cell. After removal of the cell debris by centrifugation $(20 \mathrm{~min}$ at $12,000 \times \mathrm{g}$ ), three 5 -ml aliquots of cell lysate were centrifuged for $2 \mathrm{~h}$ at $100,000 \times g$. To analyze the association of proteins with the membrane, the pelleted membranes were resuspended in $2.5 \mathrm{ml}$ of buffer, in either $10 \mathrm{mM}$ Tris- $\mathrm{HCl}(\mathrm{pH}$ 8.0) containing $500 \mathrm{mM} \mathrm{KCl}, 100 \mathrm{mM} \mathrm{Na}_{2} \mathrm{CO}_{3}(\mathrm{pH} 11.0)$, or $10 \mathrm{mM}$ Tris- $\mathrm{HCl}$ (pH 8.0) containing $1 \%$ Triton $\mathrm{X}-100$. Upon incubation for $15 \mathrm{~min}$ at $4^{\circ} \mathrm{C}$, the samples were recentrifuged for $2 \mathrm{~h}$ at $100,000 \times g$. The supernatant was collected, and the pellet of each sample was resuspended in $2.5 \mathrm{ml}$ buffer containing $10 \mathrm{mM}$ Tris- $\mathrm{HCl}(\mathrm{pH} 8.0)$ and $1 \%$ Triton $\mathrm{X}-100$, followed by an additional incubation for $15 \mathrm{~min}$ at $4^{\circ} \mathrm{C}$. Equal amounts were analyzed by sodium dodecyl sulfate (SDS)-polyacrylamide gel electrophoresis (PAGE) and Western blotting and with Tat-specific antibodies using $\mathrm{CDP}^{*}$ reagent (Tropix) detection.

Production of PspA-specific antibodies. For immunodetection of the S. lividans PspA protein, polyclonal PspA antisera were generated. This was done by immunizing rabbits with a peptide representing a predicted immunogenic epitope of the PspA protein, $\mathrm{H}_{2} \mathrm{~N}$-LDRAEDPRETLDYSC-COOH (Eurogentec), coupled to a carrier. Coupling to the hapten carrier and purification of the resulting antisera were performed according to the manufacturer's guidelines (Imject Maleimide activated immunogen conjugation kit; Pierce). The obtained antiserum was tested for its specificity by Western blotting analysis.

Construction of a PspA transposon mutant. A derivative of cosmid St6G10A carrying a Tn5062 insertion in the $p s p A$ gene (a kind gift of Paul Dyson, University of Swansea), generated using an in vitro transposition method (3), was introduced into $E$. coli $\mathrm{S}-17$ cells. In the subsequent step, $S$. lividans TK24 cells were conjugated with the cosmid-containing $E$. coli as described previously by Kieser et al. (12).

Two MRYE plates, one containing nalidixic acid $(25 \mu \mathrm{g} / \mathrm{ml})$ and apramycin $(50 \mu \mathrm{g} / \mathrm{ml})$ and the other containing nalidixic acid and kanamycin $(50 \mu \mathrm{g} / \mathrm{ml})$, were prepared. Single transconjugants were patched as a grid onto both plates (with the kanamycin plate first) and incubated for $\sim 4$ days. Transconjugants in which a double crossover had occurred (i.e., patches that were apramycin resistant and kanamycin sensitive) were identified. These patches were then taken through single-colony isolation on MRYE plates containing apramycin and nalidixic acid, and single colonies were further checked for kanamycin sensitivity before spore suspensions were made and stored at $-20^{\circ} \mathrm{C}$. Four independent $\mathrm{Apr}^{\mathrm{r}} \mathrm{Kan}^{\mathrm{s}}$ transconjugants were checked by Southern blot analysis to verify that the expected double crossover and allelic replacement had occurred in each case (results not shown).

A complemented strain was constructed by introducing plasmid pIJ486xPspA, carrying the $p s p A$ gene, into the mutant.

Promoter analysis. Expression from the $p s p A$ promoter was analyzed using the psp $A$ transposon mutant. The Tn5062 transposon contains a promoterless enhanced green fluorescent protein (eGFP) gene. Insertion of the transposon behind the promoter of the $p s p A$ gene, as done here for the creation of the mutant, allowed an analysis of the promoter activity of the disrupted gene (3).

Dynamic eGFP fluorescence measurements were done using the Infinite200 system (Tecan Group). Briefly, 24-well plates (Greiner Bio One) were inoculated with $10^{6}$ Streptomyces spores in $1 \mathrm{ml} \mathrm{NM}$ medium per well. Spores were allowed to germinate and grow at $29^{\circ} \mathrm{C}$ while shaking ( $4 \mathrm{~mm}$, orbital). After $18 \mathrm{~h}$, the

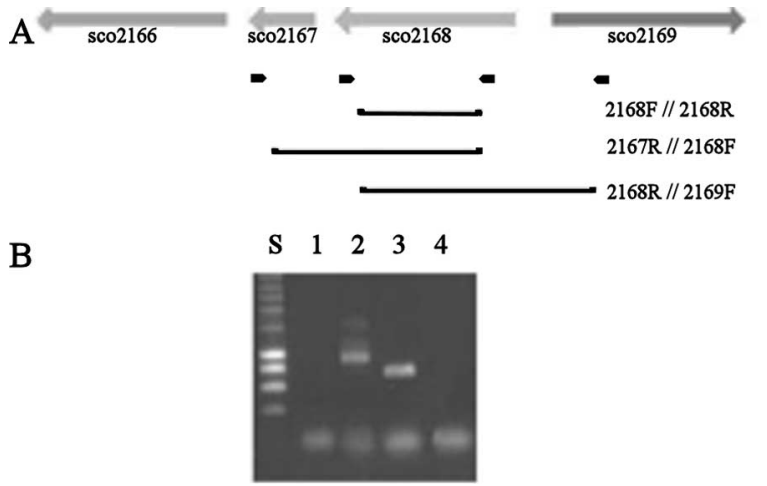

FIG. 1. RT-PCR and gene organization. (A) Schematic representation of the gene region of $p s p A$ (sco2168) showing four open reading frames. Primers used for RT-PCR analysis are indicated by arrows, and expected lengths are indicated by horizontal lines. The figure is not drawn to scale. (B) Agarose gel electrophoresis of the RT-PCR products. Smart Ladder (Eurogentec) was used as a DNA marker in lane S. Lane 1 contains the control PCR, done without reverse transcriptase, to check the absence of DNA contamination. Lanes 2, 3, and 4 show the obtained RT-PCR products for primer pairs $2167 \mathrm{R} / 2168 \mathrm{~F}, 2168 \mathrm{~F} /$ $2168 \mathrm{R}$, and $2168 \mathrm{R} / 2169 \mathrm{~F}$, respectively.

shock factor was added, and the cells were allowed to grow for another $24 \mathrm{~h}$ under the same conditions. During growth, eGFP fluorescence was measured every $2 \mathrm{~min}$.

Activity assays. Extracellular eGFP activity of $200 \mu \mathrm{l}$ spent medium was assayed by measuring fluorescence with a Fluoroskan Ascent FL fluorophotometer (Labsystems), with the excitation filter set to $485 \mathrm{~nm}$ and the emission filter set to $520 \mathrm{~nm}$.

Xylanase activity was measured with a dinitrosalicylic acid assay as described previously (6). Briefly, after $24 \mathrm{~h}$ of growth, cultures were centrifuged (10 min at $4,000 \times g$ at $4^{\circ} \mathrm{C}$ ), the obtained supernatants were diluted in the assay buffer, and the amount of reducing sugar was quantified. One unit of xylanase was defined as the amount of enzyme that produces $1 \mathrm{mg}$ reducing sugar in $10 \mathrm{~min}$ at $60^{\circ} \mathrm{C}$ from a saturated xylan solution. Values were expressed as units per mg mycelial dry weight to correct for possible differences in growth rates between the different $S$. lividans strains.

\section{RESULTS}

Transcriptional analysis of the $S$. lividans $p s p A$ gene. The transcriptional organization of genes in the neighborhood of the $\operatorname{sp} A$ gene was analyzed using the genomic sequence of a sequenced $S$. coelicolor strain as a starting point (sco numbers). Both organisms are highly similar, and work by Leblond et al. (16) revealed essentially similar genomic organizations in the two species. $p s p A$ (sco2168 homologue) appears to form an operon with the downstream-located sco2167 homologue, a gene encoding a $10-\mathrm{kDa}$ protein of unknown function. This apparent operon structure is conserved in a wide range of high-GC-content, gram-positive bacteria. Further downstream, we can find a two-component sensor kinase/response regulator pair (sco2166/sco2165 homologues), while 306 bp upstream of the $p \operatorname{sp} A$ gene, a divergently transcribed gene encoding a predicted integral membrane protein of unknown function (sco2169 homologue) is present. RT-PCR experiments were carried out on total RNA isolated from WT S. lividans TK24 to verify possible cotranscription of the sco2167 and sco2169 gene homologues with the $p s p A$ gene (Fig. 1). RT-PCR with primers $2168 \mathrm{~F}$ and $2168 \mathrm{R}$, both binding to the $p s p A$ gene, gave a 780-bp band, revealing a $p s p$ transcript. Similarly, RT-PCR 
A

—WT S. lividans $\nrightarrow$ pspA mutant $+\mathrm{pspA} \multimap-\mathrm{pspA}$ mutant

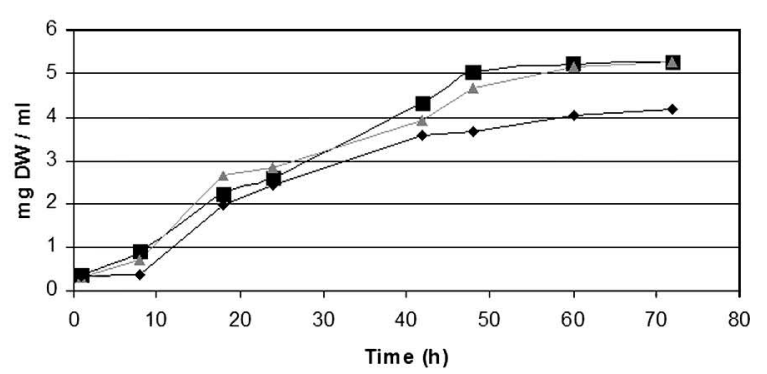

B
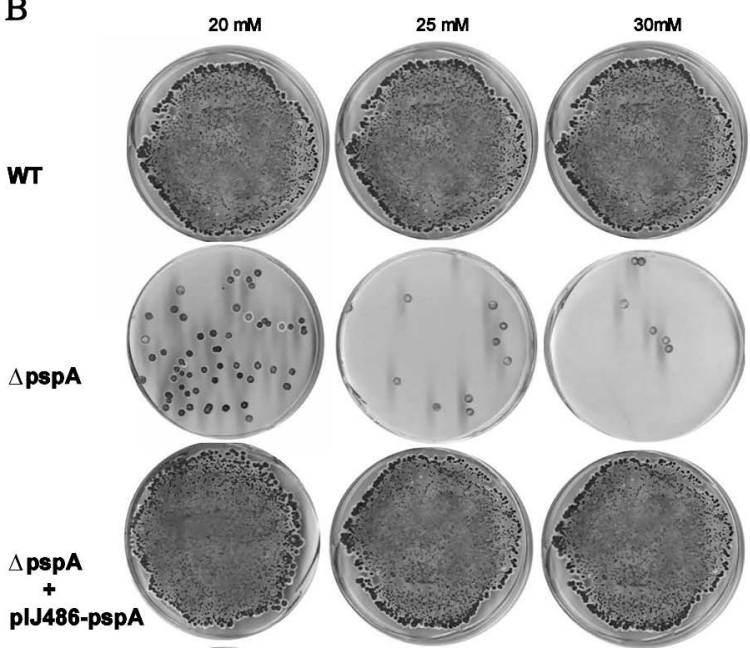

FIG. 2. PspA promotes the survival of S. lividans in the stationary phase under alkaline conditions. (A) Growth curves of WT $S$. lividans, the $p s p A$ mutant, and the complemented $p s p A$ mutant. Strains were grown in nonbuffered NB medium. DW, dry weight. (B) Survival of WT $S$. lividans, the $p \operatorname{sp} A$ mutant, and the complemented strain. The top row shows the CFU of WT $S$. lividans in 100 $\mu \mathrm{l}$ of NB medium after $12 \mathrm{~h}$ of growth in the presence of 20,25 , or $30 \mathrm{mM} \mathrm{NaOH}$. The middle and bottom rows show the results for the same experiment with the psp $A$ mutant strain and the complemented strain, respectively.

with primers $2168 \mathrm{~F}$ and $2167 \mathrm{R}$ gave a 910-bp band, indicating that $p s p A$ and the sco2167 gene homologue are cotranscribed. RT-PCR on RNase-treated samples was negative, confirming a specific, DNA-independent amplification.

$p s p A$ mutants show decreased viability in alkaline environments. To prove the importance of the PspA protein in the $S$. lividans extracytoplasmic stress response, we constructed a $p s p A$ mutant, which was subsequently subjected to a wide variety of stresses. Its growth and survival were compared to those of WT $S$. lividans. When both strains were grown in buffered NM medium, no growth difference could be observed. However, when both strains were grown in nonbuffered NB medium for 3 days, the mutant strain showed a decreased cell density in the stationary phase (Fig. 2A). The $\mathrm{pH}$ in unbuffered medium was found to be $\sim 9.2$ after $72 \mathrm{~h}$, in contrast to $\mathrm{pH} 8.2$ in buffered medium. We therefore investigated whether $p s p$ mutants could survive under alkaline conditions.

Precultures were made by growing the $S$. lividans strains in 5 $\mathrm{ml}$ phage medium for $24 \mathrm{~h}$. After $24 \mathrm{~h}$, this culture was homogenized, and $3 \mathrm{mg}$ mycelial dry weight was used to inoculate
$50 \mathrm{ml} \mathrm{NB}$ medium. The cultures were grown for $18 \mathrm{~h}$, after which $\mathrm{NaOH}$ was added to the medium in increasing concentrations from 0 to $30 \mathrm{mM}$, giving a $\mathrm{pH}$ range from an initial $\mathrm{pH}$ of 7.2 to a pH of 9.5 at $30 \mathrm{mM}$. Subsequently, these cultures were incubated for an additional $12 \mathrm{~h}$, following which aliquots of the culture were removed, serially diluted, and plated onto MRYE agar to determine the survival rate.

As shown in Fig. 2B, at higher concentrations of $\mathrm{NaOH}$, the psp $A$ mutant showed a severe decrease in viability compared to the viability of the WT. At 20 to $30 \mathrm{mM} \mathrm{NaOH}$, corresponding to a $\mathrm{pH}$ of 8.9 to 9.5 , the number of colonies of the WT strain decreased from $\sim 20,000$ to $15,000 \mathrm{CFU} / \mathrm{ml}$, whereas for the mutant, a mere 100 to $0 \mathrm{CFU} / \mathrm{ml}$ could be detected. The viability was restored to WT levels in the complemented mutant, indicating an important role for $p s p A$ in survival in alkaline environments. These results are consistent with results obtained for $E$. coli where bacteria lacking the $p s p A B C$ genes exhibited a substantial decrease in the ability to survive incubation in stationary phase under alkaline conditions $(\mathrm{pH} \mathrm{9)}$ (28).

psp $A$ mutants show increased sensitivity to extracytoplasmic stress. Following the observation that growth of the $p s p$ mutant differed from that of the WT strain when cultured under alkaline conditions, we compared the growth of the WT strain to that of the $p s p A$ mutant in the presence of other types of extracytoplasmic shock. We compared the effect of a wide variety of shocks, i.e., heat, ethanol, hyperosmotic shock, and SDS treatment, on WT $S$. lividans, the $p s p A$ mutant, and the complemented strain.

Since it has been shown that heat shock induces the psp response in $E$. coli, we hypothesized that a similar response might be seen in $S$. lividans. However, when the mutant was grown for $18 \mathrm{~h}$ and then subjected to a series of heat shocks, ranging in temperature from $37^{\circ} \mathrm{C}$ to $50^{\circ} \mathrm{C}$ and in time from 15 min to $1 \mathrm{~h}$, no difference in growth or viability could be seen for either strain (data not shown). The fact that in E. coli, temperature shock is the only shock not relying on the PspB and PspC proteins, neither of which is present in S. lividans, made this even more remarkable.

Unlike heat shock, other factors did have a significant effect on the growth of the psp mutant. Figure 3 shows that when cultures were subjected to a $5 \%$ ethanol shock, the mutant showed a very different growth curve from that of the WT. Contrary to the alkaline shock, ethanol shock did not appear to kill the cells of the $p s p A$ mutant but instead showed an aggravated growth defect compared with growths of the WT and the complemented strain.

Furthermore, when the $p s p A$ mutant was grown under hyperosmotic shock conditions, it grew to a consistently lower density than the WT (Fig. 4). The hyperosmotic shock was caused by inoculating the preculture directly into medium containing increasing $\mathrm{NaCl}$ concentrations. To make sure that the observed effect was due to hyperosmotic shock and not a consequence of ionophoric shock, the experiment was repeated with increasing concentrations of sucrose, which cannot be metabolized by $S$. lividans (12). It is important that, although not metabolized, the addition of sucrose to the medium causes more dispersed growth, making a quantitative comparison between the $\mathrm{NaCl}$ and sucrose shock conditions impossible. However, we could clearly see a growth defect in the $p s p A$ mutant 

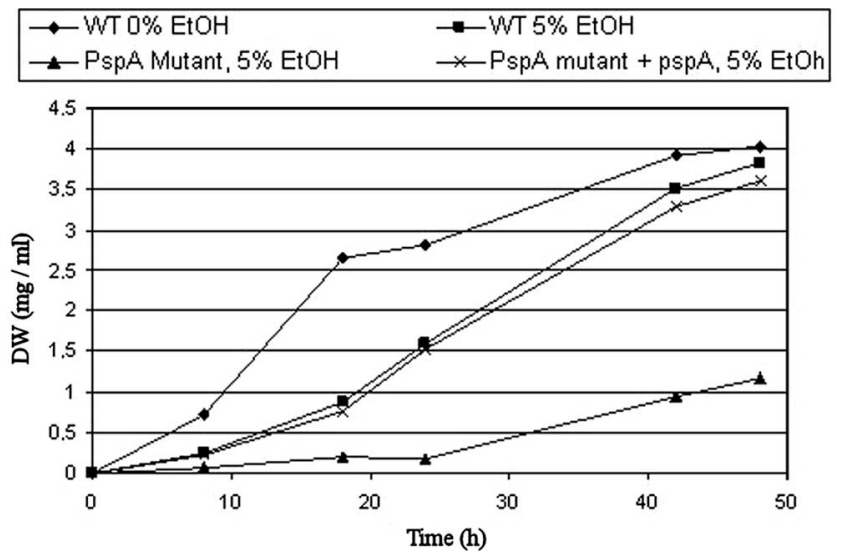

FIG. 3. PspA is important for growth in the presence of ethanol. Growth curves of WT $S$. lividans, the $p s p A$ mutant, and the complemented $p s p A$ mutant are shown. All strains were grown in NM medium supplemented with 0 or $5 \%$ ethanol $(\mathrm{EtOH})$. The $p s p A$ mutant clearly shows decreased growth in the presence of $5 \%$ ethanol. DW, dry weight.

similar to the one observed with $\mathrm{NaCl}$ when grown in the presence of 0.7 to $1 \mathrm{M}$ of sucrose (Fig. 4). All observed growth defects due to hyperosmotic shock could be restored in the complemented strain (data not shown).

Finally, the $p s p A$ mutant showed an increased sensitivity to the anionic detergent SDS. As shown in Fig. 5, the growth of the mutant was much more impaired than the growth of WT $S$. lividans at levels of SDS exceeding $0.01 \%$.

psp $A$ mutants show decreased sensitivity to bacitracin. When Bacillus subtilis cells were subjected to treatment with bacitracin, an inhibitor of cell wall biosynthesis, its $p s p A$ homologue, liaH (previously $y v q H$ ), showed a 10 -fold increase in transcription levels (18). Since B. subtilis, like $S$. lividans, has no other $p s p$ homologues, we investigated the effect of bacitracin on the $\operatorname{sp} A$ mutant.
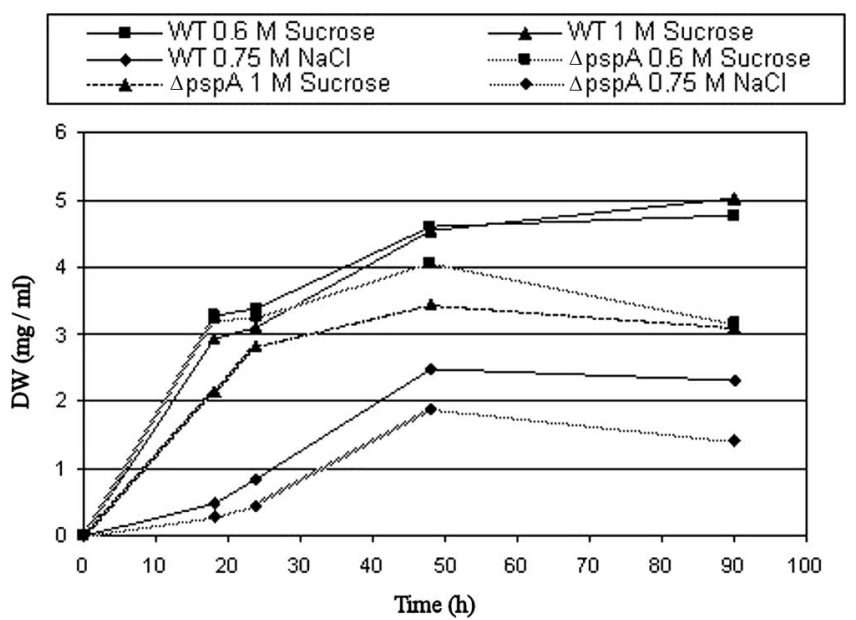

FIG. 4. PspA plays an important role in resistance to high osmolarity. Growth curves of WT $S$. lividans and the $p s p A$ mutant are shown. Both strains were grown in NM medium supplemented with $0.75 \mathrm{M}$ $\mathrm{NaCl}$ or 0.6 to $1 \mathrm{M}$ sucrose. The $p s p A$ mutant clearly shows decreased growth under conditions of hyperosmotic shock.

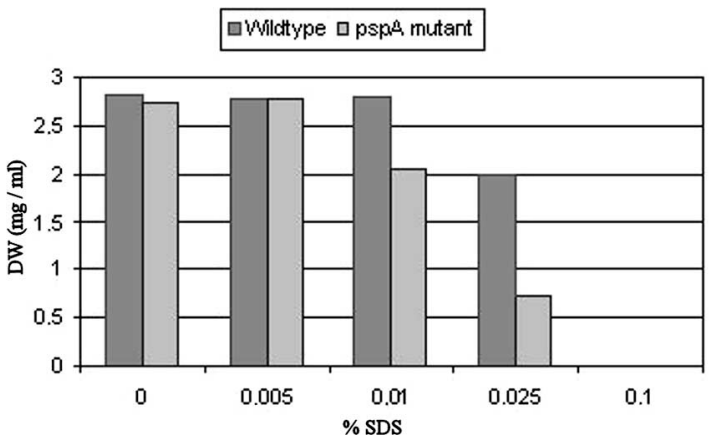

FIG. 5. PspA mutants show an increased sensitivity to SDS. Fifty milliliters of NM medium was inoculated with a WT S. lividans or $p s p A$ mutant preculture (3 mg, dry weight [DW]) and, where applicable, supplemented with different concentrations of SDS. After $24 \mathrm{~h}$ of growth, the dry weight of $1 \mathrm{ml}$ of the culture was determined. The $p s p A$ mutant clearly shows decreased viability in the presence of higher SDS concentrations.

Inhibition by bacitracin was tested in NM medium supplemented with antibiotic at concentrations ranging from 50 $\mu \mathrm{g} / \mathrm{ml}$ to $100 \mu \mathrm{g} / \mathrm{ml}$. Contrary to what we expected, the mutant strain showed an increased resistance to bacitracin. At a concentration of $50 \mu \mathrm{g} / \mathrm{ml}$, the $p s p A$ mutant showed a slower growth rate than untreated cells, but it still grew to a final dry weight of $\sim 65 \%$ of the untreated cells, whereas the growth of the WT strain was dramatically hampered at this concentration (Fig. 6).

The $p s p A$ promoter is activated by extracytoplasmic stress. The growth curves of the $S$. lividans $p s p A$ mutant under different stress conditions suggested a role for PspA in the extracytoplasmic stress response. To analyze $p s p A$ promoter activity under different stress conditions, we utilized the fusion between the promoterless eGFP gene and the $p s p A$ promoter present in the $p s p A$ mutant. This fusion allowed us to quantify the activity of the promoter by measuring the eGFP fluorescence in vivo and in real time.

Cultures were grown in NM medium for $18 \mathrm{~h}$, after which an

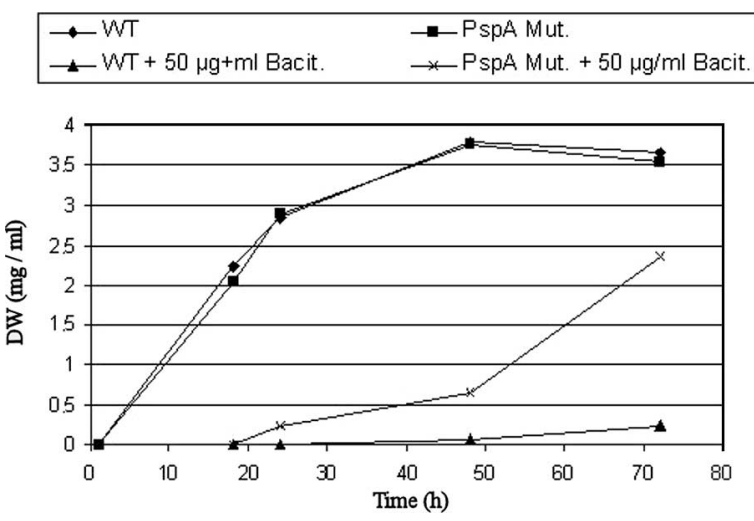

FIG. 6. PspA mutants show decreased sensitivity to bacitracin. Fifty milliliters of NB was inoculated with WT $S$. lividans or the $p s p A$ mutant (3 mg, dry weight [DW]) and, where applicable, supplemented with bacitracin. Dry weight was determined at several time points during the growth. In the presence of $50 \mu \mathrm{g} / \mathrm{ml}$ bacitracin, the WT showed almost no growth $(0.2 \mathrm{mg} / \mathrm{ml})$, whereas the $p s p A$ mutant had a final dry weight of almost $2.5 \mathrm{mg} / \mathrm{ml}$. 


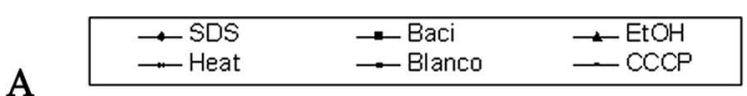

A

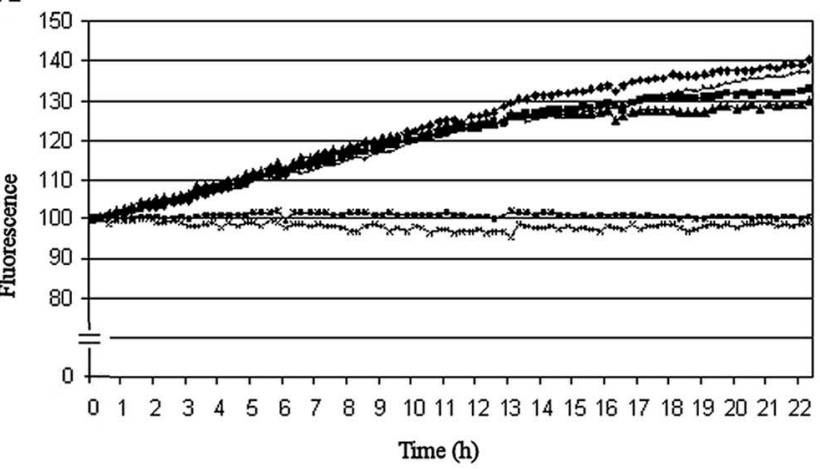

B
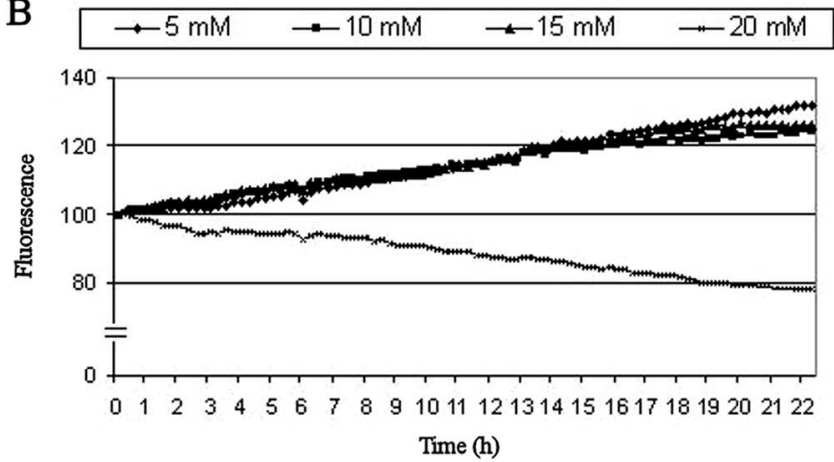

C

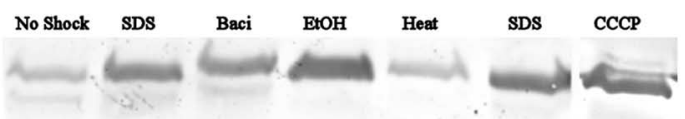

FIG. 7. $p s p A$ promoter activity assay. (A) S. lividans containing a promoterless eGFP gene under the control of the $p s p A$ promoter was grown for $18 \mathrm{~h}$, after which the medium $(t=0)$ was exposed to an extracytoplasmic shock (SDS, bacitracin [Baci], 5\% ethanol [EtOH], heat of $50^{\circ} \mathrm{C}$, or $\mathrm{CCCP}$ ). Following this shock, the fluorescence of the culture, corresponding to eGFP expression and, thus, $p s p A$ activation, was measured. Heat shock does not appear to activate the $p s p A$ promoter, but all other shocks do. (B). Cultures were shocked with various concentrations of $\mathrm{NaOH}$. (C) Validation of the promoter activity assay via Western blotting. Cell lysate fractions of S. lividans (at $t=2 \mathrm{~h}$ ) were separated by SDS-PAGE and subsequently analyzed by Western blotting and detection using PspA antiserum. PspA clearly shows an induction under all shock conditions except heat shock.

extracytoplasmic stress was added to the medium, and fluorescence was measured every $15 \mathrm{~min}$ continuously for $24 \mathrm{~h}$. Figure $7 \mathrm{~A}$ shows the resulting fluorescence patterns. Similar to the results obtained for the phenotypical characterization of the mutant, heat shock at $50^{\circ} \mathrm{C}$ did not appear to activate the $p s p A$ promoter. The other stress factors tested, including SDS, ethanol, bacitracin, and alkaline shock, all activated transcription from the $p s p A$ promoter. This activation continued for as long as the shock was present, as is obvious by the steady increase in culture fluorescence and, thus, continued eGFP gene transcription, during the entire 24-h experiment. In addition to the previous shocks, we tested whether the addition of CCCP could activate the $p s p A$ promoter. CCCP is a protonophore which dissipates the proton motive force (PMF) by conducting protons across the cytoplasmic membrane. Figure 7A shows

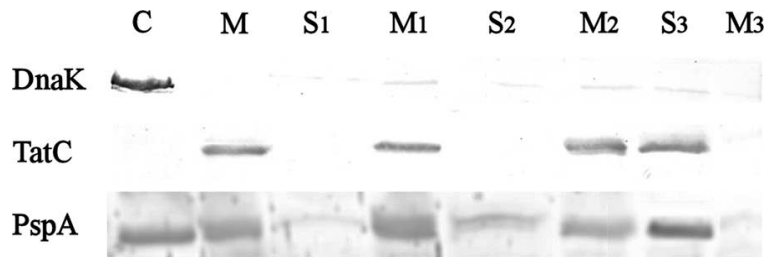

FIG. 8. Localization and membrane extraction of S. lividans PspA Equal amounts of cytoplasmic proteins $(\mathrm{C})$ and solubilized membrane proteins $(\mathrm{M})$ were loaded. In the next step, the membrane fractions were treated with $500 \mathrm{mM} \mathrm{KCl}(1)$, with $100 \mathrm{mM} \mathrm{Na}_{2} \mathrm{CO}_{3}$ (2), or with $10 \mathrm{mM}$ Tris- $\mathrm{HCl}(\mathrm{pH} 8)$ plus $1 \%$ Triton X-100 (3) and separated into solubilized proteins $(\mathrm{S})$ and the remaining membrane pellet $(\mathrm{M})$. The proteins were separated on a $12.5 \%$ SDS-PAGE gel and visualized after Western blotting using specific antisera and a chemiluminescent detection procedure. As a control, the same procedure was repeated for the cytoplasmic reporter DnaK and the integral membrane protein TatC.

that after exposure to $6 \mu \mathrm{M} \mathrm{CCCP}$, the medium fluorescence increased, indicating that the dissipation of the PMF can trigger a PspA response in S. lividans.

To investigate whether the activation of the $p s p A$ promoter depended on the severity of the membrane shock, we repeated the above-described experiment with an $\mathrm{NaOH}$ shock. Cultures grown for $18 \mathrm{~h}$ were supplemented with an increasing concentration of $\mathrm{NaOH}$ (5 to $20 \mathrm{mM}$ ), and the resulting activation of the $p s p A$ promoter was investigated (Fig. 7B). We found no difference in medium fluorescence in the $24 \mathrm{~h}$ following the administration of 5,10 , and $15 \mathrm{mM} \mathrm{NaOH}$. A higher concentration of $\mathrm{NaOH}$ led to a slow decrease in medium fluorescence, but this is most likely not linked to a decreased activation of the promoter but is instead linked with a sharp decrease in viable $S$. lividans cells under these conditions (see above). Ultimately, the $p s p A$ induction pattern obtained was validated at the protein level. Cell lysates obtained from $S$. lividans after the administration of the different extracytoplasmic shocks $(t=6 \mathrm{~h})$ were separated by SDS-PAGE and subsequently analyzed by Western blotting and detection using PspA antiserum. The obtained blot (Fig. 7C) clearly supports the promoter activation tests. These data, along with the similar activation for the other membrane shocks, point toward a single activation state of the $p s p A$ promoter independent of the severity of the shock.

Localization of the PspA protein. To determine the localization of PspA, fractionation of $S$. lividans cell lysates into a cytoplasmic fraction and a membrane fraction was performed. Both fractions were separated by SDS-PAGE, followed by Western blotting and detection using PspA antiserum. Strikingly, the PspA protein, despite being predicted to be a cytoplasmic protein, was identified both in the membrane fraction and in the cytoplasmic fraction (Fig. 8). Analysis of the films with ImageJ (National Institutes of Health) was used to estimate the fraction of membrane-localized PspA. Approximately $35 \%$ of PspA was found to reside in the membrane fraction.

Since the S. lividans PspA protein is predicted to contain no transmembrane helices, we investigated the stability of this membrane association. To this purpose, membrane fractions isolated from WT $S$. lividans were treated with high salt (500 $\mathrm{mM} \mathrm{KCl})$, sodium carbonate $(100 \mathrm{mM}, \mathrm{pH} 11)$, and detergent (Triton X-100). High salt and sodium carbonate are assumed 
to extract peripheric membrane proteins, whereas Triton $\mathrm{X}-100$ extracts integral membrane proteins.

Proteins extracted by the respective solutions and proteins remaining in the membrane after treatment were separated by SDS-PAGE, followed by Western blotting and detection with PspA antibodies. Figure 8 shows that little of the membranelocalized PspA could be extracted using high salt but that roughly $30 \%$ could be extracted using sodium carbonate $(\mathrm{pH}$ 11). The detergent Triton $\mathrm{X}-100$ extracted all PspA from the membrane.

Results of these localization and extraction experiments showed that different PspA populations are present in S. lividans cells. The first PspA population is located in the cytoplasm, while the second one behaves as peripheric membrane proteins. This peripheric membrane protein population seems to consist of two subpopulations: one containing PspA that is loosely associated with the membrane and that can be extracted with a high salt concentration and another population that seems to interact more tightly with the membrane or with proteins in the membrane and that can only be extracted with Triton X-100 treatment.

pspA mutants show secretion characteristics similar to those of WT S. lividans. Previously, we showed that the overproduction of PspA greatly increased the secretion of homologous and heterologous proteins through the Tat pathway by $S$. lividans (25). A similar effect was described previously for $E$. coli PspA (7), and in B. subtilis, it was shown that liaH is dramatically upregulated under conditions of secretion stress (8). Since PspA can play a stimulating role in protein secretion, we investigated if an absence or low levels of PspA might impair protein secretion.

Therefore, we transformed both WT and $p s p A$ mutant strains with a plasmid encoding the native Tat substrate xylanase $\mathrm{C}(x \ln C)$ or the heterologous eGFP gene fused in frame behind the $x \ln C$ signal peptide. After $24 \mathrm{~h}$ of growth in NM medium, the amount of eGFP or xylanase activity in the medium was measured. Surprisingly, the WT and the $p s p A$ mutant showed similar levels of secretion for both Tat substrates, i.e., $11.2 \pm 2.7 \mathrm{mg} / \mathrm{liter}$ and $12 \pm 2.96 \mathrm{mg} / \mathrm{liter}$ for eGFP and $1.58 \pm$ $0.27 \mathrm{U} / \mathrm{ml}$ and $1.42 \pm 0.30 \mathrm{U} / \mathrm{ml}$ for xylanase for the mutant and the WT, respectively. These results show that Tat-dependent protein secretion is not impaired in the $p \operatorname{sp} A$ mutant, indicating that Tat-dependent protein secretion does not rely on the presence of a functional PspA protein but can be enhanced by it.

\section{DISCUSSION}

The $p s p$ response in $E$. coli is known to be induced by various stress conditions such as heat shock, hyperosmotic shock, ethanol treatment, and treatment with hydrophobic solvents (reviewed in reference 5). All these shocks provoke injury to the membrane, and a unifying consequence may be the dissipation of the PMF, although this has not been tested in most cases.

It has therefore been hypothesized that the $p s p$ response plays a role in the maintenance of the PMF. PspA is thought to be the main effector protein of the response and is highly conserved in many organisms. Strikingly, most of these $p s p A$ homologues are not adjacent to any other $p s p$ gene, making it unlikely that they are part of a psp response similar to the one in the Enterobacteriaceae.

Previously, we reported that the S. lividans PspA homologue positively influences protein secretion. This positive effect was much more pronounced in Tat-dependent secretion, a process relying only on the PMF for energy, indicating that $p s p A$ in $S$. lividans may play a role in PMF maintenance similar to that of $p s p A$ in $E$. coli despite the absence of other psp genes.

In this study, we show a clear similarity between both PspA proteins, since in $S$. lividans, the $p s p A$ mutant also showed a decreased growth rate under most of the conditions tested. The strongest effect was noted for alkaline shock, which plays an important role in stationary-phase survival due to the alkalization of the medium. The viability of the $p s p A$ mutant strain quickly dropped to zero once the $\mathrm{pH}$ of the medium reached 9. Interestingly, in B. subtilis, another gram-positive bacterium containing only a $p s p A$ homologue (liaH), this gene was highly induced, as observed in a microarray analysis of the alkaline shock response (29).

Similarly to the liaH gene, $S$. lividans $p s p A$ was induced by bacitracin. The latter drug interferes with the lipid II cycle in the cytoplasmic membrane and can thus induce membrane stress. Unexpectedly, the $S$. lividans psp $A$ mutant showed an increased resistance to bacitracin.

This observation seems to contradict a possible role in the maintenance of membrane integrity or the PMF, but in $B$. subtilis, it has been shown that LiaH can play a dual role. It can be involved directly or indirectly in countering membrane stress, but additionally, it has been shown to be a negative regulator of LiaRS, a two-component system involved in bacitracin resistance (10). Homologues of LiaRS exist in $S$. lividans, and it is possible that by taking away the negative regulation, the $S$. lividans $p s p A$ mutant acquires increased bacitracin resistance.

With the notable exception of heat shock, we showed that all the conditions tested induce $p s p A$ expression. Furthermore, these conditions all cause a growth defect in the $p s p A$ mutant, highlighting the importance of PspA in the $S$. lividans membrane stress response.

In the E. coli model, PspA is presumed to be the main effector protein, with PspB and PspC as putative, membranelocated sensor proteins. The absence of homologues of these sensor proteins in $S$. lividans makes it remarkable that its $p s p A$ responses resemble those in $E$. coli so much. It is, however, important that although most inducing stimuli in E. coli depend entirely on PspB and PspC (19), some stress conditions rely on only one of the $p s p B$ and $p s p C$ genes or do not require PspB or PspC at all (27). It is therefore possible that $S$. lividans PspA directly responds to stress conditions, although it seems more likely that an as-yet-unidentified membrane protein acts as a sensor in the process. In B. subtilis, the LiaI membrane protein has been proposed to be putative sensor for $\mathrm{LiaH}$, although experimental data supporting this hypothesis are still lacking.

The localization of the PspA protein in S. lividans also supports the existence of an as-yet-unidentified membrane partner. PspA was found in both the cytoplasmic $(\sim 65 \%)$ and membrane $(\sim 35 \%)$ fractions. Since PspA contains no sequences characteristic of integral membrane proteins, it is likely that the protein interacts with either a protein in the 
membrane or the phospholipids directly. In E. coli, which contains $\sim 50 \%$ membrane-associated PspA, it was assumed that this association was due to an interaction with the integral membrane proteins PspB and PspC (1). However, a very recent article (14) described that purified PspA in its oligomeric state can also bind to phosphatidylserine- and phosphatidylglycerol-containing liposomes in the absence of PspB and PspC. Most likely, both the lipid bilayer and some proteins associated with it can contribute to PspA localization in S. lividans.

Kobayashi et al. (14) also found previously that PspA had the ability to suppress proton leakage of damaged membranes and that PspB and PspC were not prerequisites for this function. Their proposed model, with PspA oligomers interacting with phosphatidylserine/phosphatidylglycerol in the membrane and, as such, altering the physical properties of the membrane to suppress proton leakage, could be applied to other organisms independent of the presence of other Psp proteins.

The observation that Tat-dependent secretion is not impaired in the $p s p A$ mutant might seem to be contradictory at first. We hypothesize that while PspA is involved in PMF maintenance and repair under stress conditions, there is probably little influence on the PMF in unshocked cells. In E. coli, Kleerebezem et al. (13) previously showed that the membrane potential in a $p s p A$ mutant and that in the WT are approximately the same. Furthermore, DeLisa and colleagues (7) showed that $p \operatorname{sp} A$ is not induced by the overexpression of the Tat substrate sufI or by overexpressing Tat substrates that were previously shown to be misfolded. An increase in PspA synthesis was caused only by mutations that abolished export nearly completely. These data indicate that PspA is not an absolute requirement for Tat secretion and that other factors contributing to the regulation of the PMF might be able to compensate for the lack of PspA in the mutant strain when there is overexpression of a Tat substrate.

The fact that PspA homologues are found in many bacterial species combined with the data presented in this study indicate that even when no PspBC homologues are present, PspA plays an important role in the membrane stress response in bacteria.

\section{ACKNOWLEDGMENTS}

K.V. is a research fellow of the Institute for the Promotion of Innovation by Science and Technology in Flanders. This research was supported by grants G.0389.05 from the Fonds voor Wetenschappelijk Onderzoek Vlaanderen and QLK3-LSHG-CT-2007-037586 from the European Union.

\section{REFERENCES}

1. Adams, H., W. Teertstra, J. Demmers, R. Boesten, and J. Tommassen. 2003 Interactions between phage-shock proteins in Escherichia coli. J. Bacteriol. 185:1174-1180. doi:10.1128/JB.185.4.1174-1180.2003.

2. Anné J., L. Van Mellaert, and H. Eyssen. 1990. Optimum conditions for efficient transformation of Streptomyces venezuelae protoplasts. Appl. Microbiol. Biotechnol. 32:431-435. doi:10.1007/BF00903778.

3. Bishop, A., S. Fielding, P. Dyson, and P. Herron. 2004. Systematic insertional mutagenesis of a streptomycete genome: a link between osmoadaptation and antibiotic production. Genome Res. 14:893-900. doi:10.1101/gr.1710304.

4. Brissette, J. L., M. Russel, L. Weiner, and P. Model. 1990. Phage shock protein, a stress protein of Escherichia coli. Proc. Natl. Acad. Sci. USA 87:862-866. doi:10.1073/pnas.87.3.862.

5. Darwin, A. J. 2005. The phage-shock-protein response. Mol. Microbiol. 57:621-628. doi:10.1111/j.1365-2958.2005.04694.x.

6. De Keersmaeker, S., L. Van Mellaert, E. Lammertyn, K. Vrancken, J. Anne, and N. Geukens. 2005. Functional analysis of TatA and TatB in Streptomyces lividans. Biochem. Biophys. Res. Commun. 335:973-982. doi:10.1016 j.bbrc. 2005.07.165.
7. DeLisa, M. P., P. Lee, T. Palmer, and G. Georgiou. 2004. Phage shock protein PspA of Escherichia coli relieves saturation of protein export via the Tat pathway. J. Bacteriol. 186:366-373. doi:10.1128/JB.186.2.366-373.2004.

8. Hyyrylainen, H. L., M. Sarvas, and V. P. Kontinen. 2005. Transcriptome analysis of the secretion stress response of Bacillus subtilis. Appl. Microbiol. Biotechnol. 67:389-396. doi:10.1007/s00253-005-1898-1.

9. Jones, S. E., L. J. Lloyd, K. K. Tan, and M. Buck. 2003. Secretion defects that activate the phage shock response of Escherichia coli. J. Bacteriol. 185:67076711. doi:10.1128/JB.185.22.6707-6711.2003.

10. Jordan, S., A. Junker, J. D. Helmann, and T. Mascher. 2006. Regulation of LiaRS-dependent gene expression in Bacillus subtilis: identification of inhibitor proteins, regulator binding sites, and target genes of a conserved cell envelope stress-sensing two-component system. J. Bacteriol. 188:5153-5166. doi:10.1128/JB.00310-06.

11. Jovanovic, G., L. Weiner, and P. Model. 1996. Identification, nucleotide sequence, and characterization of PspF, the transcriptional activator of the Escherichia coli stress-induced psp operon. J. Bacteriol. 178:1936-1945.

12. Kieser, T., M. J. Bibb, M. J. Buttner, K. F. Chater, and D. A. Hopwood. 2000. Practical Streptomyces genetics. John Innes Foundation, Norwich, United Kingdom.

13. Kleerebezem, M., W. Crielaard, and J. Tommassen. 1996. Involvement of stress protein PspA (phage shock protein A) of Escherichia coli in maintenance of the protonmotive force under stress conditions. EMBO J. 15:162-171.

14. Kobayashi, R., T. Suzuki, and M. Yoshida. 2007. Escherichia coli phageshock protein A (PspA) binds to membrane phospholipids and repairs proton leakage of the damaged membranes. Mol. Microbiol. 66:100-109. doi: 10.1111/j.1365-2958.2007.05893.x.

15. Korn, F., B. Weingärtner, and H. J. Kutzner. 1978. Genetics of the Actinomycetales, p. 251-270. In E. Freechsen, I. Tarnak, and J. H. Thumin (ed.), A study of twenty actinophages: morphology, serological relationship and host range. Fisher G., Stuttgart, Germany.

16. Leblond, P., M. Redenbach, and J. Cullum. 1993. Physical map of the Streptomyces lividans 66 genome and comparison with that of the related strain Streptomyces coelicolor A3(2). J. Bacteriol. 175:3422-3429.

17. Lloyd, L. J., S. E. Jones, G. Jovanovic, P. Gyaneshwar, M. D. Rolfe, A. Thompson, J. C. Hinton, and M. Buck. 2004. Identification of a new member of the phage shock protein response in Escherichia coli, the phage shock protein G (PspG). J. Biol. Chem. 279:55707-55714. doi:10.1074/jbc.M408994200.

18. Mascher, T., N. G. Margulis, T. Wang, R. W. Ye, and J. D. Helmann. 2003. Cell wall stress responses in Bacillus subtilis: the regulatory network of the bacitracin stimulon. Mol. Microbiol. 50:1591-1604. doi:10.1046/j.13652958.2003.03786.x

19. Model, P., G. Jovanovic, and J. Dworkin. 1997. The Escherichia coli phageshock-protein (psp) operon. Mol. Microbiol. 24:255-261. doi:10.1046/j.13652958.1997.3481712.x.

20. Pozidis, C., E. Lammertyn, A. S. Politou, J. Anné, A. S. Tsiftsoglou, G. Sianidis, and A. Economou. 2001. Protein secretion biotechnology using Streptomyces lividans: large-scale production of functional trimeric tumor necrosis factor alpha. Biotechnol. Bioeng. 72:611-619. doi:10.1002/1097-0290 (20010320)72:6<611::AID-BIT1026>3.0.CO;2-0.

21. Rowley, G., M. Spector, J. Kormanec, and M. Roberts. 2006. Pushing the envelope: extracytoplasmic stress responses in bacterial pathogens. Nat. Rev. Microbiol. 4:383-394. doi:10.1038/nrmicro1394.

22. Sianidis, G., C. Pozidis, F. Becker, K. Vrancken, C. Sjoeholm, S. Karamanou, M. Takamiya-Wik, L. van Mellaert, T. Schaefer, J. Anné, and A. Economou. 2006. Functional large-scale production of a novel Jonesia sp. xyloglucanase by heterologous secretion from Streptomyces lividans. J. Biotechnol. 121:498-507. doi:10.1016/j.jbiotec.2005.08.002.

23. Van Dessel, W., L. Van Mellaert, N. Geukens, E. Lammertyn, and J. Anné. 2004. Isolation of high quality RNA from Streptomyces. J. Microbiol. Methods 58:135-137. doi:10.1016/j.mimet.2004.03.015.

24. Van Mellaert, L., C. Dillen, P. Proost, E. Sablon, R. Deleys, A. Van Broekhoven, H. Heremans, J. Van Damme, H. Eyssen, and J. Anné. 1994. Efficient secretion of biologically active mouse tumor necrosis factor alpha by Streptomyces lividans. Gene 150:153-158. doi:10.1016/0378-1119(94)90876-1.

25. Vrancken, K., S. De Keersmaeker, N. Geukens, E. Lammertyn, J. Anné, and L. Van Mellaert. 2007. pspA overexpression in Streptomyces lividans improves both Sec- and Tat-dependent protein secretion. Appl. Microbiol. Biotechnol. 73:1150-1157. doi:10.1007/s00253-006-0571-7.

26. Watve, M. G., R. Tickoo, M. M. Jog, and B. D. Bhole. 2001. How many antibiotics are produced by the genus Streptomyces? Arch. Microbiol. 176: 386-390. doi:10.1007/s002030100345.

27. Weiner, L., J. L. Brissette, and P. Model. 1991. Stress-induced expression of the Escherichia coli phage shock protein operon is dependent on sigma 54 and modulated by positive and negative feedback mechanisms. Genes Dev. 5:1912-1923. doi:10.1101/gad.5.10.1912.

28. Weiner, L., and P. Model. 1994. Role of an Escherichia coli stress-response operon in stationary-phase survival. Proc. Natl. Acad. Sci. USA 91:21912195. doi:10.1073/pnas.91.6.2191.

29. Wiegert, T., G. Homuth, S. Versteeg, and W. Schumann. 2001. Alkaline shock induces the Bacillus subtilis $\sigma^{\mathrm{W}}$ regulon. Mol. Microbiol. 41:59-71. doi:10.1046/j.1365-2958.2001.02489.x. 